\title{
Immunisation and allergy in children and adults
}

\author{
A case-based approach
}

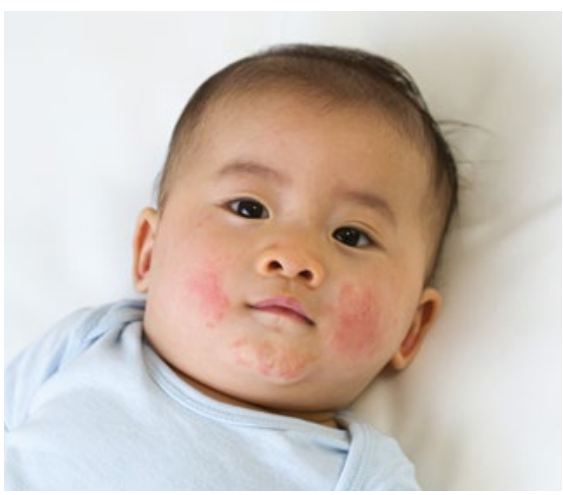

CPD

Abigail Cheung, Kirsten P Perrett

\section{Background}

Adverse events following immunisation (AEFIs) are often attributed to vaccine hypersensitivity. However, many of these possible allergic reactions are unlikely to be confirmed as true vaccine allergies but rather coincidental symptoms that may mimic an allergic reaction.

\section{Objective}

By using case studies, the authors show the variation of presentations of potential hypersensitivity AEFIs. Case studies are used to illustrate a general approach to investigation and management of these reactions.

\section{Discussion}

AEFIs are commonly seen in general practice, and it can often be difficult to differentiate between the underlying mechanisms. It is important to be able to identify a potential hypersensitivity reaction so that it can be reported to the appropriate local pharmacovigilance system and patients can be reviewed by immunisation specialists to evaluate, investigate and manage future immunisations where required.
ADVERSE EVENTS following immunisation (AEFIs) in Australia have been reported at a rate of 16.9 per 100,000 population. The most commonly reported reactions include injection site reaction (ISR; 34\%), pyrexia (15\%), rash (15\%), vomiting $(8 \%)$, headache $(6 \%)$ and pain $(6 \%)$, with anaphylaxis comprising $0.9 \%$ of AEFIs reported. ${ }^{1}$

One study in Victoria found that suspected immediate hypersensitivity reactions (non-specific rash, urticaria, angioedema, allergic reaction [generalised] or anaphylaxis occurring within 60 minutes of immunisation) accounted for almost a quarter $(23.5 \%)$ of all AEFIs reported in preschool-aged children. The rate of overall incidence of suspected immunoglobulin (Ig) E-mediated reaction was two per 100,000 doses, and the anaphylaxis rate was 0.13 per 100,000 doses. $^{2}$

True vaccine allergy, where a person is investigated and found to have positive skin testing or a repeated hypersensitivity reaction on challenge/revaccination, is rare; it is found in $<10 \%$ of children investigated and/or challenged in a specialist allergy unit following a potential vaccine hypersensitivity reaction. ${ }^{3} \mathrm{~A}$ vaccination reaction should be referred to as an AEFI until it can be categorised. Reporting these as 'allergic reactions' can be a deterrent for further vaccination, resulting in incomplete immunisation coverage.
Many AEFI reactions are thought to represent hypersensitivity reactions but are often due to other mechanisms, as outlined in Table 1. Many of these are rare and out of the scope of this discussion; however, almost all warrant immunisation specialist review. In the cases that follow, the authors demonstrate common presentations of suspected hypersensitivity reactions in children and adults, and discuss the underlying mechanisms and management.

\section{CASE 1: SALLY}

Sally, aged four years, presented two days after receiving her routine diphtheria/ tetanus/pertussis (DTPa) four-year-old booster immunisation. She had pain and swelling around her injection site. The pain and swelling had commenced approximately 24 hours after the vaccination was administered (Figure 1). She was systemically well.

Pain, redness and swelling at an injection site is a common, expected side effect that usually occurs within 24 hours post-immunisation; it is seen following up to $34 \%$ of injections. ${ }^{1}$ Mild ISRs may occur after any vaccine and are secondary to non-specific inflammation due to the injection of foreign materials or the injection itself. The majority of 
ISRs are small and do not limit activity. No further action is required; however, symptomatic relief may include oral analgesia and cool compress.

An ISR is classified as severe if it involves the entire limb between two joints, also known as extensive limb swelling. This usually occurs within 24-72 hours after vaccine administration and spontaneously resolves, usually within a week. ${ }^{4}$ Symptomatic relief is generally recommended, including

\section{Table 1. Synopsis of potential immune-mediated reactions to vaccines ${ }^{10}$}

\begin{tabular}{ll}
\hline Immune-mediated reaction & Frequent clinical manifestation \\
\hline $\begin{array}{l}\text { Immunoglobulin (Ig) } \\
\text { E-mediated }\end{array}$ & $\begin{array}{l}\text { Urticaria, angioedema, rhinoconjunctivitis, } \\
\text { bronchospasm, gastrointestinal disorders (diarrhoea, } \\
\text { abdominal cramping, vomiting), anaphylaxis }\end{array}$ \\
\hline Immune complex (IgG) & Vasculitis, myocarditis \\
\hline T-cell mediated & Maculopapular exanthema, eczema, acute generalised \\
exanthematous pustulosis, erythema multiforme
\end{tabular}

Reproduced from Chung EH, Vaccine allergies, Clin Exp Vaccine Res 2014 Jan;3(1):50-57, doi: 10.7774/ cevr.2014.3.1.5, licensed under CC BY-NC 3.0.
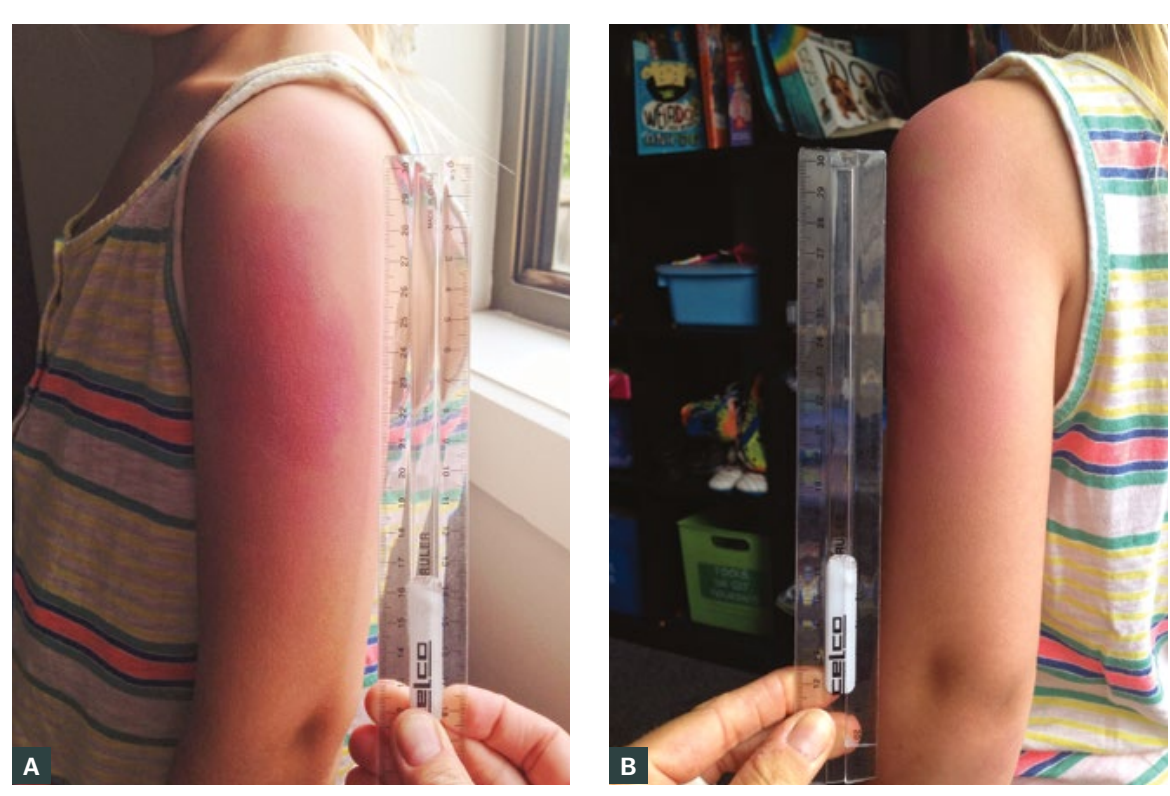

Figure 1. Injection site reaction ${ }^{17}$

A. Photograph looking directly onto the limb; B. Photograph showing the profile of the arm from behind Reproduced with permission from McGuire R, Photographing a severe local reaction, Parkville, Vic: MVEC, 2018, Available at https://mvec.mcri.edu.au/immunisation-references/photographing-a-severe-localreaction [Accessed 31 July 2020].

cool compress and analgesia if the child remains systemically well. A severe ISR is not a contraindication for further immunisations, which can be given again in a primary care setting.

ISRs are particularly common after repeated DTPa-containing vaccines, with approximately $20 \%$ of children reporting an ISR and $2 \%$ reporting a severe ISR. ${ }^{5}$ ISRs after influenza vaccination are also commonly seen, occurring in $21.2 \%$ of patients after dose one and $6 \%$ of patients after dose two; many of these reactions are mild. ${ }^{6}$ ISRs can be seen with any vaccination, with incidences reported between $5 \%$ and $30 \%$, dependent on the type of vaccination. ${ }^{7}$

Severe ISRs can be commonly mistaken for cellulitis. However, previous studies have shown swelling of both subcutaneous tissue and muscle, suggesting angioedema rather than cellulitis. ${ }^{8}$ Indeed, bacterial cellulitis post-immunisation is very rare, and antibiotics are usually not required.

\section{CASE 2: USHI}

Ushi, aged 12 months, presented with fever and a generalised non-urticarial rash. He had received his routine 12-month immunisations (ie measles/ mumps/rubella [MMR], 13-valent pneumococcal and meningococcal ACWY vaccines) six days prior to presentation. He was well prior to the immunisations being administered but had developed malaise after five days, with fever and rash developing the morning of presentation.

There is a need to distinguish between non-allergic systemic reactions and potential vaccine hypersensitivity reactions. Systemic reactions may consist of fever and other non-specific symptoms such as malaise, myalgias or headache. Skin rashes including urticaria or angioedema may also present as part of a systemic reaction. These do not conclusively indicate an allergic cause, particularly if they are delayed from the time of immunisation. While the underlying mechanisms for systemic reactions are unknown, it is likely that 
they occur as a result of non-specific activation of the immune system.

In this case, the delay between vaccination and onset of reaction indicated that it was unlikely to be secondary to a hypersensitivity (IgE-mediated) reaction to the vaccine. The delayed nature of the fever and rash reaction six days post-immunisation would be more consistent with a reaction to the live-attenuated MMR vaccine, which is typically seen after 5-12 days. A fever, which may be $>39.4^{\circ} \mathrm{C}$, occurs in up to $15 \%$ of MMR vaccine recipients and can last 2-3 days. ${ }^{9}$ A systemic reaction following inactive vaccinations would be expected within the first few days after vaccination.

Given that this was not a vaccine hypersensitivity reaction, Ushi is not at increased risk of allergic reaction or anaphylaxis with future immunisations. Even though the next routine immunisations at 18 months of age include the same MMR antigens, these would still be able to be administered in the appropriate setting with a standard observation period of at least 15 minutes as per the guidelines of the Advisory Committee on Immunisation Practices. ${ }^{7}$ He would not need any preceding investigation. In fact, adverse events are much less common after the second dose of MMR or MMR in combination with varicella vaccine than after the first dose. ${ }^{7}$

\section{CASE 3: SAANV}

Saanvi, aged two years, was brought to see you for her annual influenza immunisation. She had no significant medical history and had been well. Her examination was normal, and the influenza vaccine was administered as per standard protocols. Within 10 minutes, Saanvi developed generalised urticaria to her arms and torso but remained systemically well.

All vaccines have the potential to cause IgE-mediated reactions. Almost all of the individual vaccine components may be considered as a possible allergic trigger, which may include active immunising antigens, conjugating agents, preservatives, stabilisers, antimicrobial agents, adjuvants and culture media. ${ }^{10}$ With increasing numbers of vaccines administered, hypersensitivity reactions are more commonly reported, with the majority of these being mild. ${ }^{1}$ The most immediate and potentially severe reactions are type I hypersensitivity reactions, which are IgE-mediated. These typically occur within minutes of the immunisation, and generally within an hour of exposure; however, delayed reactions do rarely occur.

In this case, immediate generalised urticaria post-immunisation may be suggestive of a type I hypersensitivity reaction. It is important to review patients for any signs of anaphylaxis to help stratify further management. Saanvi only had dermatological manifestations, without any features of a severe reaction. She would therefore not meet the criteria for anaphylaxis. Nevertheless, referral to a specialist immunisation unit for assessment would still be recommended. Investigation and management protocols tend to vary; however, one general approach and management algorithm is shown in Figure $2 .{ }^{3}$ For children with a history of mild potential hypersensitivity reaction only, these authors recommend a supervised in-hospital provocation test (challenge) dose when the relevant vaccine is next required. The index vaccine challenge may be administered as a split dose (eg 10\%, then $90 \%$, with one hour of observation between doses). If this challenge is negative (non-reactive), future vaccines can be administered in the community with standard observation.

CASE 4: GOSIA

Gosia, aged 12 years, presented for her routine year 7 (aged 12-13 years) immunisations. She was well but appeared nervous during the consultation. The immunisation was administered, and within a minute, Gosia became pale and complained of difficulty breathing before collapsing. On examination, her observations were normal and her chest remained clear. Oxygen was applied, and she spontaneously regained consciousness within a few minutes and was subsequently monitored in hospital with no further intervention.

It can often be difficult to discern between anxiety, syncope and potential vaccine hypersensitivity. Vasovagal syncope post-immunisation is relatively common, particularly in the adolescent group, with a female preponderance. ${ }^{11}$ In the Australian population, syncope has been reported to affect up to $10 \%$ of adolescents in association with the human papillomavirus (HPV) immunisation. ${ }^{12}$ Tonic or clonic movements are also commonly reported in syncopal episodes, secondary to anoxia.

Factors that may help to discriminate between a vasovagal episode and a potential hypersensitivity (anaphylactic) reaction are listed in Table 2.

In Gosia's case, there were many factors that may create confusion regarding a possible allergic reaction, including the complaints of difficulty breathing. In the authors' clinical experience, somatic complaints are common in vasovagal syncope and are likely related to underlying anxiety. For future immunisations, measures for the prevention of syncope should be undertaken. These include monitoring for any presyncopal signs and symptoms at the time of immunisation, and advising the patient to ensure they are well hydrated to maintain blood volume, and to sit or lie down during and after the immunisation (raising legs if needed until free of symptoms).

\section{CASE 5: BRIDGET}

Bridget, aged 12 years, presented with a history of a severe reaction after her routine year 7 immunisations a few days prior. She reported that she had been well prior to the immunisation and had received the HPV and booster DTPa vaccines at school. Within five minutes, Bridget had developed generalised erythema, tightness in the chest with wheeze and dizziness. She was administered a dose of intramuscular adrenaline with good effect and sent to hospital by ambulance but did not require any further treatment. 


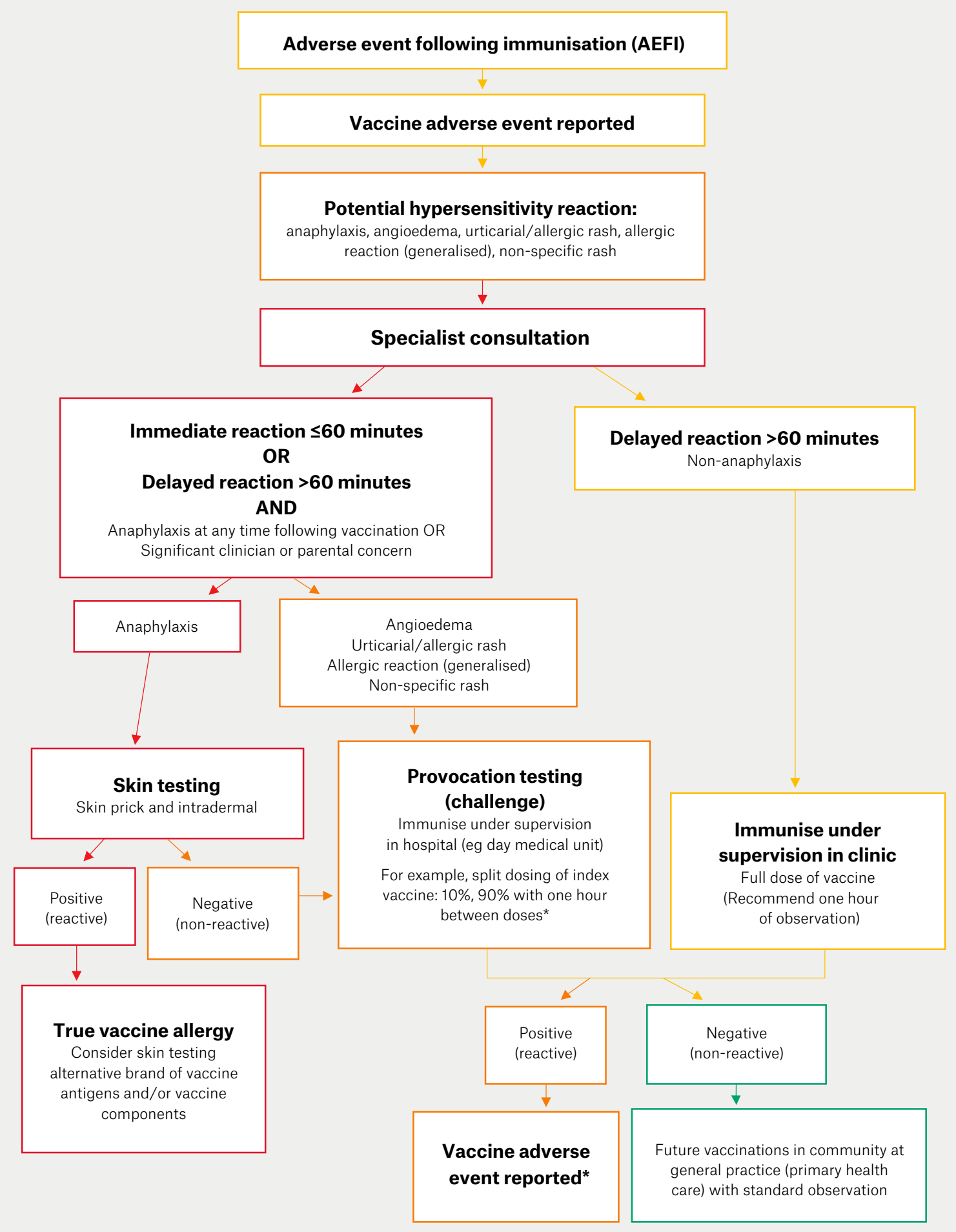

Figure 2. Suggested algorithm for suspected hypersensitivity reaction to vaccine ${ }^{3}$

*If suspected immunoglobulin E-mediated reaction at 10\% dose, seek specialist guidance prior to proceeding with further doses Reproduced with permission from Cheung A, Choo S, Perrett KP, Vaccine allergy? Skin testing and challenge at a tertiary pediatric hospital in Melbourne, Australia, J Allergy Clin Immunol Pract 2019;7(5):1541-49, doi: 10.1016/j.jaip.2019.01.025. 
Systemic severe allergic reactions are rare but, obviously, important. Anaphylaxis is set apart from other hypersensitivity or allergic reactions by the sudden onset, rapid progression and simultaneous involvement of several $(\geq 2)$ organ systems. There are multiple criteria systems that help to define and stratify the risk of anaphylaxis. One commonly used system is the Brighton Collaboration Criteria. ${ }^{13}$ However, it is important to note that in some patients, the clinical picture may not be complete. Where there is uncertainty about the diagnosis, a referral for assessment by a specialist immunisation service should be made. IgE-mediated reactions generally occur soon after vaccination, usually within the first hour.
However, it is important to note that anaphylactic reactions may occur outside of this timeframe; ${ }^{13}$ therefore, regardless of timing, any reactions consistent with anaphylaxis should be referred.

In Bridget's case, her symptoms are consistent with an anaphylactic reaction, fulfilling level 1 Brighton Collaboration Criteria. As such, she should be referred to a specialist immunisation service for further investigation and guidance for further vaccines.

Serum mast cell tryptase (MCT) levels are useful as a retrospective marker of anaphylaxis, although their use for vaccine-associated anaphylaxis has not been established. An acute rise in serum tryptase post-reaction may help to identify anaphylaxis. Hence, it is useful to perform an MCT test within two hours after suspected anaphylaxis, with a serum baseline tryptase level measured at least 48 hours post-reaction. ${ }^{4}$

Specific IgE tests to vaccine components have low predictive capacity and are not routinely recommended for evaluation. ${ }^{4}$ Skin testing (skin prick and intradermal testing) can be useful for evaluating severe vaccine reactions and will often help to guide further vaccine challenges under immunisation specialist care.

As Bridget received two different immunisations, it would be important for her to undergo skin testing to help identify which of these may have triggered her

Table 2. Clinical features that may help differentiate between a vasovagal episode and anaphylaxis ${ }^{7}$

\begin{tabular}{|c|c|c|}
\hline Clinical features & Vasovagal episode & Anaphylaxis \\
\hline $\begin{array}{l}\text { Cardiovascular } \\
\text { symptoms or signs }\end{array}$ & $\begin{array}{l}\text { - Bradycardia } \\
\text { - Weak/absent peripheral pulse } \\
\text { - Strong carotid pulse } \\
\text { - Hypotension - usually transient and } \\
\text { corrects in supine position } \\
\text { - Loss of consciousness - improves once in } \\
\text { supine or head-down position }\end{array}$ & $\begin{array}{l}\text { - Tachycardia } \\
\text { - Weak/absent carotid pulse } \\
\text { - Hypotension - sustained and no improvement without specific } \\
\text { treatment (note: in infants and young children, limpness and } \\
\text { pallor are signs of hypotension) } \\
\text { - Loss of consciousness - no improvement once in supine or } \\
\text { head-down position }\end{array}$ \\
\hline Skin symptoms or signs & $\begin{array}{l}\text { - Generalised pallor } \\
\text { - Cool, clammy skin }\end{array}$ & $\begin{array}{l}\text { - Pruritus (skin itchiness) } \\
\text { - Generalised skin erythema (redness) } \\
\text { - Urticaria (weals) } \\
\text { - Angioedema (localised or general swelling of the deeper layers } \\
\text { of the skin or subcutaneous tissues) }\end{array}$ \\
\hline $\begin{array}{l}\text { Gastrointestinal } \\
\text { symptoms or signs }\end{array}$ & Nausea or vomiting & $\begin{array}{l}\text { - Abdominal cramps } \\
\text { - Diarrhoea } \\
\text { - Nausea or vomiting }\end{array}$ \\
\hline
\end{tabular}


reaction. In hospital, she had a negative skin test to the booster DTPa vaccine but a positive skin test to the HPV vaccine. She was subsequently also skin tested to an alternative brand of HPV vaccine, which was negative. For her second HPV vaccine dose, the alternative brand was administered under supervision in hospital by split dosing, which was well tolerated. In general, anaphylaxis to a previous dose of a vaccine is a contraindication to receiving the same vaccine and the patient should be referred for immunisation specialist evaluation.

\section{CASE 6: KOSTAS}

Kostas, aged 24 years, presented for an influenza vaccination, which had been recommended due to his asthma. His medical history included an egg allergy with previous anaphylaxis. Kostas had not received the influenza immunisation previously, and his general practitioner was concerned about administering it in the primary care setting.

The current influenza vaccines in Australia are derived from influenza virus grown in hens' eggs and may potentially contain minute traces of egg protein (ovalbumin). There have been previous case reports of anaphylaxis to influenza vaccine in individuals with egg allergies; ${ }^{14}$ however, this was when the amounts of egg protein were much higher than in current vaccines.
In a review of influenza immunisation and egg allergy, there were no severe reactions after the immunisation. ${ }^{15}$ Mild side effects such as local itch, urticaria, throat irritation, wheeze or abdominal pain have been observed, but not anaphylaxis. There was also no correlation between preceding allergy testing with the vaccine and the outcome of adverse reaction. ${ }^{16}$ Current international guidelines, including those published by the Australian Society of Clinical Immunology and Allergy (ASCIA),${ }^{14}$ recommend that individuals with egg allergies (including those with egg anaphylaxis) can safely receive seasonal influenza vaccines. Guidelines specifically recommend vaccines containing $<1 \mu \mathrm{g} / \mathrm{dose}$ of egg ovalbumin, which includes all current influenza vaccines licensed in Australia in 2020. The vaccine can be administered as a single dose in a community immunisation clinic (which may or may not have direct medical practitioner supervision) followed by the standard 15-minute observation period. It is important to note that although egg allergy does not increase the risk of anaphylaxis to the influenza vaccine, there is an independent risk of anaphylaxis with each immunisation; therefore, vaccines should always be administered in facilities with staff able to recognise and treat anaphylaxis.

The vaccines in the current National Immunisation Program, including the
MMR vaccine, may be given to any person with a food allergy, even those with food-induced anaphylaxis. ${ }^{14}$ It is, however, important to note that yellow fever and $Q$ fever vaccines contain higher amounts of residual egg protein, and individuals with egg allergies who require these vaccines should be referred for immunisation specialist review.

\section{AEFI reporting services}

Each state and territory has a local pharmacovigilance AEFI-reporting service within their health department (Table 3), which collaborates with and provides de-identified data to the Therapeutic Goods Administration. In Victoria and Western Australia, the Adverse Events Following Immunisation - Clinical Assessment Network (AEFI-CAN) bridges the link between health department surveillance reporting and clinical assessment and management of people following serious or unexpected AEFIs. The AEFI-CAN vaccine safety clinics (and vaccine safety clinics in other states and territories) are able to assess and manage potential allergic reactions, which may include immunisation provider and/or patient advice and, if required, expert clinical consultation regarding the event and future immunisations. Table 3 lists regional AEFI reporting services, which will provide details for the local vaccine safety clinic as needed.

\section{Table 3. Adverse event reporting}

\begin{tabular}{llll}
\hline State/territory & Reporting service & Telephone & Website \\
\hline Australian Capital Territory & ACT Health Department & 0262052300 & www.health.act.gov.au/our-services/immunisation \\
\hline New South Wales & Local Public Health Unit & 1300066055 & www.health.nsw.gov.au/immunisation \\
\hline Northern Territory & NT Department of Health & 0889228044 & https://health.nt.gov.au \\
\hline Queensland & Queensland Health & 0733289888 & www.health.qld.gov.au/cdcg/index/adverse \\
\hline South Australia & SA Department of Health & 1300232272 & www.sahealth.sa.gov.au \\
\hline Tasmania & Direct to Therapeutic Goods & 1800044114 & www.tga.gov.au \\
\hline Victoria & Administration & & \\
\hline Western Australia & SAEFVIC & 1300882924 & www.aefican.org.au \\
\hline
\end{tabular}




\section{Conclusion}

As the number of vaccines in the Australian National Immunisation Program schedule increase, so too will the number of reported AEFIs. There are a variety of underlying mechanisms and for many of these AEFIs, it can often be difficult to determine a cause. However, any event felt to be significant and all potential hypersensitivity reactions following immunisation with symptoms such as non-specific rash, urticaria, angioedema or anaphylaxis should be reported to the appropriate local pharmacovigilance service.

\section{Key points}

- AEFIs are often attributed to vaccine hypersensitivity.

- True vaccine hypersensitivity is rare; however, many AEFIs may present similarly and can be difficult to distinguish.

- It is important to report all potential hypersensitivity AEFIs to the appropriate local pharmacovigilance services.

\section{Authors}

Abigail Cheung MBBS, FRACP, Paediatric Allergist \& Immunologist, Department of Allergy \& Immunology Women's and Children's Hospital, SA; Paediatric Allergist \& Immunologist, Department of Allergy \& Immunology, Flinders Medical Centre, SA Kirsten P Perrett MBBS, FRACP, PhD, Consultant Paediatrician, Departments of Allergy \& Immunology and General Medicine, The Royal Children's Hospital, Vic; Group Leader, Population Allergy Research Group, Murdoch Children's Research Institute, Vic; Honorary Principal Fellow, Department of Paediatrics, The University of Melbourne, Vic. kirsten.perrett@mcri.edu.au

Competing interests: None.

Funding: None.

Provenance and peer review: Commissioned, externally peer reviewed.

\section{Acknowledgements}

This work was supported by the Victorian Government's Operational Infrastructure Support Program. KPP is supported by a Melbourne Children's Clinician Scientist Fellowship.

The authors had full access to all relevant data in this study, and supporting sources had no involvement in data analysis and interpretation, or in the writing of the article.

\section{References}

1. Dey A, Wang $H$, Quinn $H$, et al. Surveillance of adverse events following immunisation in Australia: Annual report, 2018. Commun Dis Intell (2018) 2020;44. doi: 10.33321/cdi.2020.44.12.
2. Baxter CM, Clothier HJ, Perrett KP. Potential immediate hypersensitivity reactions following immunization in preschool aged children in Victoria, Australia. Hum Vaccin Immunother 2018;14(8):2088-92. doi: 10.1080/21645515.2018.1460293.

3. Cheung A, Choo S, Perrett KP. Vaccine allergy? Skin testing and challenge at a tertiary pediatric hospital in Melbourne, Australia. J Allergy Clin Immunol Pract 2019;7(5):1541-49. doi: 10.1016/j. jaip.2019.01.025.

4. Nilsson L, Brockow K, Alm J, et al. Vaccination and allergy: EAACI position paper, practical aspects. Pediatr Allergy Immunol 2017;28(7):628-40. doi: 10.1111/pai.12762.

5. Halperin SA, Scheifele D, Mills E, et al. Nature, evolution, and appraisal of adverse events and antibody response associated with the fifth consecutive dose of a five-component acellular pertussis-based combination vaccine. Vaccine 2003;21(19-20):2298-306. doi: 10.1016/s0264410x(03)00173-7.

6. Wood NJ, Blyth CC, Willis GA, et al. The safety of seasonal influenza vaccines in Australian children in 2013. Med J Aust 2014;201(10):596-600. doi: 10.5694/mja13.00097.

7. Australian Technical Advisory Group on Immunisation (ATAGI). Australian immunisation handbook. Canberra, ACT: DoH, 2018. Available at https://immunisationhandbook.health.gov.au [Accessed 31 July 2020].

8. Marshall HS, Gold MS, Gent R, et al. Ultrasound examination of extensive limb swelling reactions after diphtheria-tetanus-acellular pertussis or reduced-antigen content diphtheriatetanus-acellular pertussis immunization in preschool-aged children. Pediatrics 2006;118(4):1501-09. doi: 10.1542/peds.20052890.

9. McLean HQ, Fiebelkorn AP, Temte JL, Wallace GS. Prevention of measles, rubella, congenital rubella syndrome, and mumps, 2013: Summary recommendations of the Advisory Committee on Immunization Practices (ACIP). MMWR Morb Mortal Wkly Rep 2013;62(4):1-34.

10. Chung EH. Vaccine allergies. Clin Exp Vaccine Res 2014 Jan;3(1):50-57. doi: 10.7774/cevr.2014.3.1.50.

11. Braun MM, Patriarca PA, Ellenberg SS. Syncope after immunization. Arch Pediatr Adolesc Med 1997;151(3):255-59. doi: 10.1001/ archpedi.1997.02170400041007.

12. Crawford NW, Hodgson K, Gold M, Buttery J, Wood N. Adverse events following HPV immunization in Australia: Establishment of a clinical network. Hum Vaccin Immunother 2016;12(10):2662-65. doi: 10.1080/21645515.2016.1192737.

13. Rüggeberg JU, Gold MS, Bayas JM, et al. Anaphylaxis: Case definition and guidelines for data collection, analysis, and presentation of immunization safety data. Vaccine 2007;25(41):5675-84. doi: 10.1016/j. vaccine.2007.02.064.

14. Australasian Society of Clinical Immunology and Allergy. ASCIA guidelines - Vaccination of the egg-allergic individual. Balgowlah, NSW: ASCIA, 2017. Available at www.allergy.org.au/hp/ papers/vaccination-of-the-egg-allergic-individual [Accessed 31 July 2020].

15. Kelso JM. Administering influenza vaccine to egg-allergic persons. Expert Rev Vaccines 2014;13(8):1049-57. doi: 10.1586/14760584.2014.933079.
16. Kelso JM, Greenhawt MJ, Li JT. Update on influenza vaccination of egg allergic patients. Ann Allergy Asthma Immunol 2013;111(4):301-02. doi: 10.1016/j.anai.2013.07.030.

17. McGuire R. Photographing a severe local reaction. Parkville, Vic: MVEC, 2018. Available at https:// mvec.mcri.edu.au/immunisation-references/ photographing-a-severe-local-reaction [Accessed 31 July 2020]. 